\title{
APLICAÇÃO DA LUZ INFRAVERMELHA (IV) NO ESTUDO PETROGRÁFICO E DE INCLUSÕES FLUIDAS EM MINERAIS OPACOS
}

\author{
FRANCISCO JAVIER RIOS, KAZUO FUZIKAWA, JAMES VIEIRA ALVES \& JOSÉ MARQUES CORREIA NEVES
} ABSTRACT Fluid inclusion study in opaque minerals using IR microscopes and cameras is known since I984. Using IR diodes and lamps
coupled to petrographic microscope and Fluid Inc. heating/freezing stage, microscopy and microthermometry in sphalerite, wolframite, and
hematite have been performed at the CDTN fluid inclusion laboratory. The obtained results and constraints to the used technique are presented.

Keywords: fluid inclusion, opaque minerals, infrared (IR)

\begin{abstract}
INTRODUÇÃo Boa parte dos óxidos e a maior parte dos sulfetos, wolframatos, teluretos, etc, comportam-se como opacos quando examinados em lâminas petrográficas utilizando-se o espectro da luz visível $(0,35-0,75 \mu \mathrm{m})$. Entretanto, nos últimos 15 anos, alguns laboratórios conseguiram estudar feições internas e inclusões fluidas em minerais notadamente opacos, como a wolframita, pirita, cassiterita, estibinita, enargita, tennantita, tetraedrita, bournonita e esfalerita (Campbell et al. 1984 Campbell \& Robinson Cook 1987, e mais recentemente Richards \& Kerrich 1993, Lüders 1996, Lüders \& Ziemann 1999, Bailly et al. 2000). Nesses estudos foram utilizados microscópios de infravermelho (modelos Olympus BHSM e Research Devices) e câmaras de infravermelho (Electrophysics e Hamamatsu) que permitem operar na faixa de comprimento de onda entre 0,75 e $2,2 \mu \mathrm{m}$ (Lüders \& Ziemann 1999). Quando submetidos à incidência da luz infravermelha, esses minerais tornam-se transparentes sendo possível observar zoneamentos, linhas de crescimento e inclusões sólidas e fluidas.
\end{abstract}

ABSORÇÃO IV EM MINERAIS OPACOS Os minerais escuros (sob luz transmitida) comportam-se como opacos em decorrência da absorção de fotons durante as transições eletrónicas nos orbitais moleculares. Assim, a opacidade de vários sulfetos, arseniatos, etc, com relação a luz visível, é devida a transições band-gap (Richards \& Kerrich 1993). O band gap, num mineral semicondutor, é a energia mínima necessária para excitar um elétron desde o estado (atômico) ligado na rede (a banda de valência) até um estado livre (deslocado), no qual o elétron torna-se livre para se mover (Campbell et al. 1984). Entretanto, quando a energia dos fotons é menor do que a mínima requerida para essa transição eletrónica a luz não é absorvida e o mineral comporta-se como transparente nesse comprimento de onda (Richards \& Kerrich 1993). O valor mínimo de energia para absorção (band gap energy) é atingido (para a maior parte dos sulfetos e sulfosais) na faixa do infravermelho (Burns, in Campbell et al. 1984), sendo que a radiação IV possui maior comprimento de onda (e menor energia) do que a luz visível (Mancano \& Campbell 1995). Portanto, é necessário utilizar uma energia menor que a do gap (no caso luz IV) para que (porções) do mineral opaco se comportem como transparentes. Entretanto, a substituição dentro da estrutura cristalina por impurezas de valência contrastante muda a configuração eletrônica do mineral, diminuindo a energia do band gap, resultando num decréscimo da transparência (Richards \& Kerrich 1993).

MÉTODO UTILIZADO Em conseqüência da não disponibilidade de um microscópio de infravermelho foi necessário elaborar uma fonte emissora equivalente, para ser acoplada em dois microscópios Leica DMRXP e Leitz Ortholux II Pol-BK. Para isso, nos laboratórios da CT2-CDTN, foi construída uma fonte (resistor) de corrente variável e alta sensibilidade, que opera com leds (diodo emissor de luz (infravermelho ou visível) e lâmpadas de infravermelho. Nas várias tentativas foram utilizados leds convencionais e da Laser Monitoring ( $\lambda$ entre 0,7 e $1,8 \mu \mathrm{m}$ ) e uma lâmpada de $50 \mathrm{~W}$ (tipo iodine tungsten, que opera na faixa de infravermelho). Para visualizar as imagens de IV foi utilizada uma câmara de alta resolução modelo CC-IR da Computar-Japan e um monitor-video printer Sony Trinitron. A câmara opera na faixa de $\lambda$ que abarca desde a luz visível até $1,2 \mu \mathrm{m}$ (infravermelho). Para os estudos microtermométricos com este tipo de fontes foi utilizada uma platina US Fluid Inc. A platina Chaixmeca, devido às características da sua construção, não permitiu desenvolver estudos utilizando esse método.

AMOSTRAS ESTUDADAS Para os testes com IV em minerais opacos foram selecionadas amostras de wolframita, hematita e esfalerita. A seguir serão exemplificados os resultados obtidos nos dois primeiros minerais.

Wolframita (Amostras da Jazida de Pedra Preta, Carajás, Pará). O estudo por luz IV permitiu observar o zoneamento desses cristais (Fig. $1 \mathrm{~A}$ e 1B) e verificar a ocorrência de inclusões fluidas (IF) primárias e secundárias. As IF primárias são geralmente tubulares e bifásicas $(\mathrm{L}+$ $\mathrm{V})$, com dimensões entre 5 e $15 \mu \mathrm{m}$ (Fig. 1E). As IF secundárias aparecem dispostas ao longo de planos de microfraturas, apresentam formas irregulares e são mormente monofásicas $(L)$. Durante os estudos microtermométricos das IF primárias foi possível determinar a temperatura de fusão de clatratos (em torno de $+5,5^{\circ} \mathrm{C}$ ) e de homogeneização total (entre $270 \mathrm{e} 330^{\circ} \mathrm{C}$ ). Esses valores de temperatura são coincidentes com os anteriormente obtidos por geotermometria isotópica de oxigênio (par mineral quartzo - wolframita) que indicou temperaturas em torno de $320^{\circ} \mathrm{C}$ para a precipitação do tungstato (Rios et al. 1998).

Hematita (Amostras da Mina de Águas Claras - MG e da Mina da Conceição, Itabira - MG). Nos cristais estudados, a microscopia de infravermelho permitiu detectar a presença de maclas polissintéticas bem desenvolvidas (Fig.1C e 1D). Nas amostras de Conceição foi possível observar, ainda, inclusões fluidas primárias, mormente bifásicas, de dimensões variáveis (entre 5 e $25 \mu \mathrm{m}$, Fig.1E), algumas com morfologia de cristal negativo. Os estudos microtermométricos preliminares permitiram determinar que o mineral precipitou a partir de fluidos de média - alta salinidade (equivalente a $19 \%$ em peso de $\mathrm{NaCl}$ ) e temperatura de homogeneização entre 150 e $200^{\circ} \mathrm{C}$. Uma abordagem mais abrangente dos dados obtidos nessas hematitas será apresentada em Rosière et al. (em preparação).

CONCLUSÕES A microscopia de infravermelho, nas condições em que está sendo desenvolvida, é uma metodologia demorada e custosa. Para obter os resultados apresentados foi necessário confeccionar um número elevado de lâminas especiais para IV. Entretanto, poucas foram aproveitadas em decorrência de que os minerais opacos raramente apresentam boa transparência. O objetivo do Laboratório de Inclusões Fluidas (LIF), a médio prazo, é estudar sulfetos, principalmente pirita, para o qual será necessária a aquisição de uma câmara de IV de maior definição, já que a atual permite operar numa faixa muito limitada do espectro do IV. Concluindo, com os equipamentos existentes no LIF do CDTN, estudos petrográficos e microtermométicos de cristais de wolframita, esfalerita e algumas variedades de hematita podem ser efetuados.

Agradecimentos À equipe da CT2 (CDTN), especialmente Eden Cristiano Costa (responsável pela construção dos emissores de IV), Leandro H. F. de Andrade, Pedro Lana Gastelois e Maximiliano Delany Martins. A Max Passos Ferreira pelo empréstimo da lâmpada de infravermelho. Aos colegas Carlos Alberto Rosière (IGC-UFMG, pela cessão das amostras da Mina de Conceição, e Volker Lüders (Potsdam, Alemanha) e Andrew Campbell (New Mexico-USA) e a dois revisores anônimos da RBG pelas sugestões feitas. 

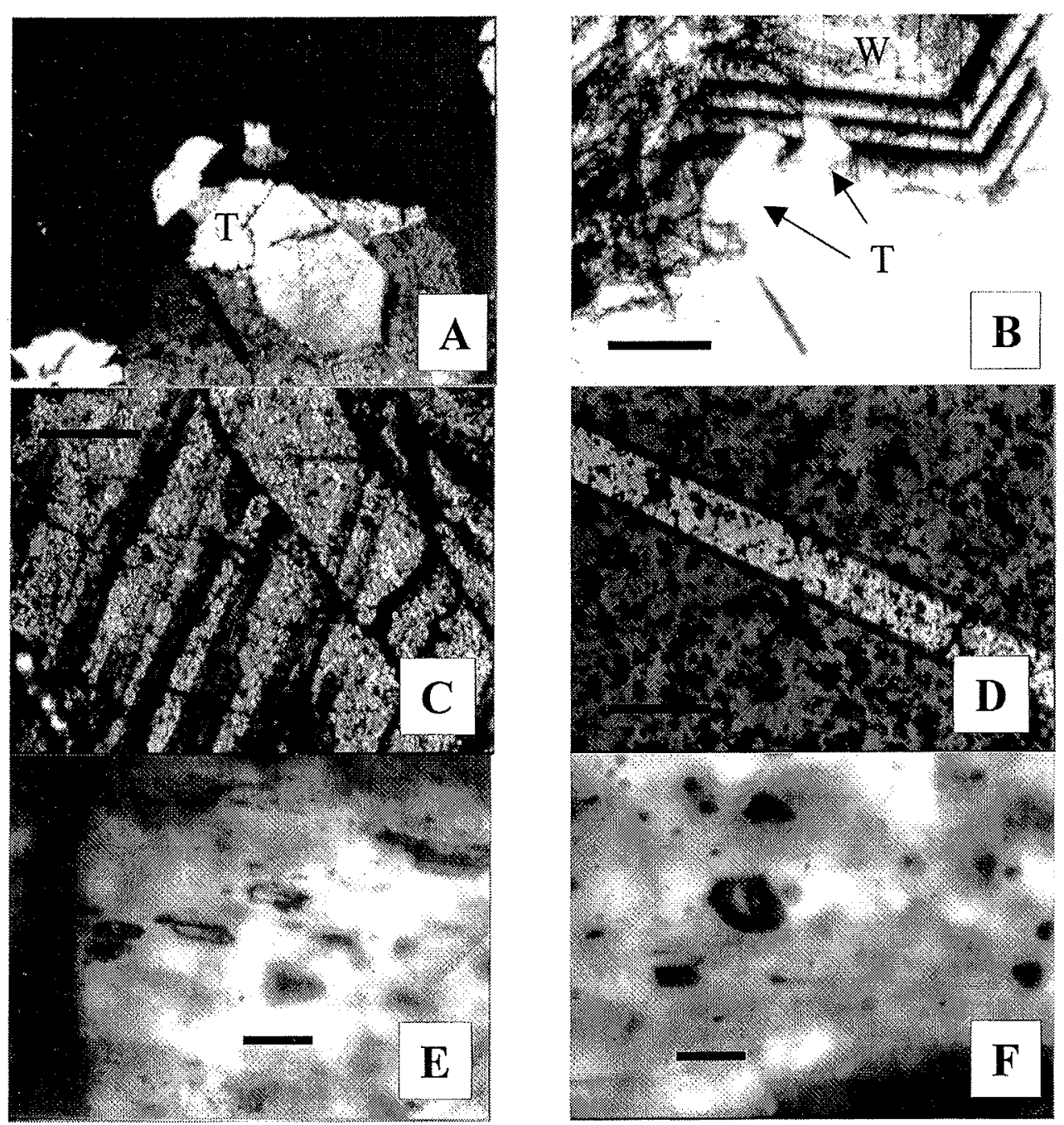

Figura I - Fotomicrografias: (A) Cristais de wolframita (em preto) e topázio (T) sob luz transmitida. (B) Mesma seção, sob luz IV. Notar zoneamentos na wolframita (W).A inclusão de topázio na wolframita (ver Fig JA) corta o zoneamento desta sendo, portanto, posterior a ela. Barra: $0.5 \mathrm{~mm}$. (C) Cristal de hematita (Mina da Conceição, Itabira - MG) sob luz IV mostrando maclas polissintéticas. Barra: 0,5 mm. (D) Cristal de hematita (Mina de Águas Claras, Nova Lima, MG) sob luz IV com macla polissintética bem definida. (E) Inclusões fluidas bifásicas em wolframita da Jazida de Pedra Preta, PA. Barra: 10 pm. (F) Inclusões fluidas bifásicas en hematita da Mina da Conceição, Itabira, MG. Barra: $10 \mu \mathrm{m}$.

\section{Referências}

Bailly L., Bouchot V., Bény C., Milési J. P. 2000. Fluid inclusion study of stibinite using infrared microscopy: an example from the Brouzils Antimony Deposit (Vendée. Armorican Massif, France) Econ. Geol., 95:221-226.

Campbell A.R Hackbarth C. Plumlee G.S., Petersen U. 1984. Internal features of ore minerals seen with the infrared microscope. Econ. Geol., 79:1387-1392.

Campbell A.R \& Robinson Cook S. 1987. Infrared fluid inclusion microthermometry on coexing wolframite and quartz. Econ Geol, 82:1640-1645.

Liders $V .1996$. Contribution on infrared microscopy to fluid inclusion studies in some opaque minerals (wolframite, stibnite, bournonite): metallogenic implications. Econ. Geol 91:1462-1468

Lüders V. \& Ziemann M. 1999. Possibilities and limitis of infrared light microthermometry applied to studies of pyrite-hosted fluid inclusions. Chentcal Geol., 154:169-178. Mancano D. P. \& Campbell A. R 1995. Microthermometry of enargite hosted fluid inclusions from Lepanto, Phillipines, high sulfidation Cu-Aul deposit. Geochim. ef Cosmochim Acta, 59:3909-3916.
Richards J.P \& Kerich R. 1993. Observations of zoning and fluid inclusions in pyrite using . Econ. Geol., 88:716-723.

Rios F J Villat R infrared light microscope. Econ. Geol., 88. 1998. Isótopos de oxigên., Villas R. N. N., Fuzikawa K., Sial A. N., Mariano G. 1998. Isótopos de oxigêPedra Preta, Sul do Pará. Rev. Bras: Geoc., 28:253-256.

Manuscrito NB-22

Recebido em 20 de junho de 2000 Revisão dos autores em 30 de julho de 2000 Revisão aceita em 15 de agosto de 2000 